observed pulsating radio-sources with a very short and steady period, they interpreted it as emission of beamed radiation from a rotating neutron star - a "pulsar". Since then, about 300 pulsars have been discovered, with periods ranging from $33 \mathrm{~ms}$ to $5 \mathrm{~s}$. The rotation period increases with time at the rate predicted by Pacini (1967) who assumed that the star has a dipolar magnetic field, of strength $\sim 10^{12}$ gauss at the surface of the star, and that it is shedding angular momentum by steadily emitting magnetic-dipole electromagnetic radiation. His calculation assumed that the star is surrounded by vacuum but we now know that it has a magnetosphere. Nevertheless, the decelerating torque on the star, though due to other processes, is of the same magnitude as given by the vacuum dipole formula.

The problem of the structure of the pulsar magnetosphere is also very complex, and has not yet been satisfactorily solved - except in perhaps the least exciting case, that of inactive pulsars. Active pulsars, which emit incredibly coherent radio radiation, and in some cases also optical light, $X$-rays and $\gamma$-rays, must be accelerating particles to relativistic energies. In present models, the acceleration occurs in "gaps" which are empty regions close to the polar cap of the star, or along the magnetic field lines connected to the poles, where DC electric fields, parallel to the magnetic field, can be maintained for more or less long times. The voltage drop across such a gap can attain $10^{13} \mathrm{~V}$. Electrons, accelerated by the electric field along the curved field lines, emit $\gamma$-rays of curvature radiation (just like relativistic electrons spiralling around field lines emit synchrotron radiation). The $\gamma$-rays in the strong magnetic field produce electronpositron pairs, increasing the supply of particles to be accelerated. It is possible that the fast electrons streaming along field lines tend to "gather in bunches" which emit coherent radio-radiation. Other models assume that the high energy radiation is emitted at the light cylinder, i.e. at the distance from the star axis at which the rigid body rotation velocity becomes equal to the velocity of light.

\section{Binary Systems}

Neutron stars have now been observed in another context notably when they are part of a binary system. Matter from the stellar companion keeps falling on the surface of the neutron star and, as it hits the surface, its considerable kinetic energy is transformed into heat, giving rise to the emission of large fluxes of $X$-rays. Because the infalling matter has so much angular momentum, it forms a disk in the orbital plane, and slowly spirals in. Of the 100 strong galactic $\mathrm{X}$-ray sources known, 16 at least involve a neutron star in a binary system. The rotation period of the neutron stars, in this case, decreases with time, as the neutron star is spun up by the infalling matter.

In the spectrum of one of these systems, $\mathrm{Her} \mathrm{XI}$, a line is clearly visible at $58 \mathrm{keV}$. It is attributed to cyclotron resonance and has led to the first measurement of the surface magnetic field of a neutron star; the value obtained is in excellent agreement with the early predictions. Recently, a similar line has been observed in another type of system; namely in the spectrum of transient sources, called "gamma-ray burst sources", which only shine for a few seconds. Such $\gamma$-ray burst sources are probably also neutron stars, which are accreting matter - but, it could be mentioned, only from the interstellar medium. Other, softer, transient sources, the "X-ray bursters", are also believed to be associated with neutron stars; the accreted matter can attain such high temperatures and den- sities that thermonuclear reactions take place on the star surface.

Finally, we should mention the famous "binary pulsar", where a pulsar is separated from its companion (probably another neutron star) by only $\sim 1$ solar radius. After several years of careful observation of this system with the Arecibo telescope, Taylor and his collaborators have found that the orbital period of the system $(7.75 \mathrm{~h})$ increases at a rate of $10^{-4} \mathrm{~s} /$ an : this result is exactly as predicted by the theory of general relativity. Thus, neutron stars have led to the first (though indirect) detection of gravitational radiation, and have become a powerful tool for the testing of post-newtonian gravitation theories.

These are just some of the topics which will be discussed at the Symposium on Neutron Stars, at the Istanbul Conference.

\title{
Heavy Ion Collisions
}

\section{N. Vinh Mau, Orsay}

One of the most active domains of nuclear physics is that of heavy ion physics, where a large amount of new information has been obtained in the recent past and more can be expected as new and powerful accelerators come into operation. From the experimental data already available, simple and general trends characterizing heavy ion collisions, can be extracted. These suggest new fundamental concepts and help in the construction of models through which phenomena can be studied. The field of heavy ion physics is rich indeed, and provides us with the means for producing new nuclei with large numbers of neutrons, nuclear matter of very high density or very high angular momentum, new reactions such as deep inelastic processes, etc...

The study of a system comprising two heavy ions in collision involves the treatment of a finite, strongly interacting fermion system which is far from equilibrium. It is clear that such a complex system is not large enough to be described entirely by classical statistical mechanical methods, and yet is too far from equilibrium to be covered by the usual microscopic methods of many body theory which are very successful at, or near, equilibrium. However, it can be hoped that both concepts will be helpful in understanding the main features of ion-ion systems as they can exhibit some properties which are typical of a classical infinite system, and others more relevant to a quantal finite system.

\section{Theoretical Treatment}

As the microscopic one body mean field picture (Hartree-Fock) of a nucleus at equilibrium, and the small amplitude vibrations picture (RPA) of collective nuclear states near equilibrium, have been so successful in describing many nuclear properties, it is natural to attempt a description of ion-ion collisions by extending these pictures. This is achieved by the time dependent Hartree-Fock (TDHF) method, which describes the system as a "one body system" in which each independent nucleon interacts with a time-dependent mean field. The solutions of the static Hartree-Fock equations, which describe so well the nuclei at equilibrium, are special solutions of the TDHF equations. On the other hand the RPA picture of collective vibrations around equilibrium, can be derived as the small amplitude limit of the TDHF approach, which provides us with a microscopic description of heavy ion collisions. This relates the complex phenomena to the nuclear ground state and near equilibrium properties.

Once the TDHF problem has been solved, information on the collision: energy loss, mass and angular momentum transfer etc... are extracted, which can be compared to experimental data. The results should help us to derive or justify, macroscopic models of ion-ion collisions which are more tractable as they retain the mean field aspect of the collision but contain only 
few dynamical variables, in contrast to a microscopic model. Comparison of the results of TDHF models with those of macroscopic models will then be very fruitful in providing a physical insight into the mechanisms involved in the collision, which is difficult to obtain directly from TDHF calculations.

The TDHF equations have been solved, despite their enormous technical difficulty, for a large range of cases going from collisions between s-d shell nuclei to collision of uranium on uranium. One may extract from the results, some general and qualitative features. Fusion cross sections are generally well reproduced, which indicates that at least some features of dissipative processes are correctly taken into account in a mean field description. This is confirmed by the calculation of average kinetic energy loss as a function of deflection angle. However, for low impact parameter and sufficiently high bombarding energy, TDHF always indicates a transparency of each ion to the other. Despite a large kinetic energy loss, the emerging fragments are very similar to the colliding ions. This prediction does not seem to be supported by experiment and is an indication of the deficiency of a simple mean field approximation. In general then, one can say that TDHF seems to predict well the average properties but does not reproduce the dispersion around them.

These conclusions have motivated recent work which tries to understand more deeply what is included in the TDHF approach and the limits of its applicability. It is also searching for modifications that should be made to the TDHF equations. Two important questions suggest themselves:

(1) what is the mechanism that generates the dissipation in TDHF and how does it relate to the classical dissipative formalism which makes a distinction between one and two body viscosity?

(2) what are the effects of two body collisions on TDHF solutions?

The purpose of the Istanbul nuclear physics symposium is to give a general review of the experimental situation as it concerns heavy ion collisions, together with the most recent developments of mean field models and their extrapolations.

\section{Continuation}

Further texts in the series will be published in the February issue of Europhysics News, notably:

High Energy and Particle Physics, 1981: Where Do We Stand? G. Preparata; Trends in Quantum Electronics,

D.S. Chemla;

Physics Education in Secondary Schools in

Europe, G. Born:

Physics and Society at Istanbul,

D.H. Parkinson.

\section{Recent Developments in Solid State Physics}

\section{J.T. Devreese, Antwerp}

Considerable progress has been realized in condensed matter physics over the past few years as new techniques have become available on both the experimental and theoretical side. On the experimental side one could name, inter alia, the use of synchrotron radiation, the application of angular resolved photoemission spectroscopy (ARPES), or the extended X-ray absorption fine structure technique (EXAFS). On the theoretical side, the concepts and techniques of the renormalization group, or the ever increasing power of computational techniques to calculate electronic structures come to mind. Also the architecture of materials has developed; e.g. using molecular beam epitaxy, superlattices are prepared with composition and smoothness controlled on the scale of atomic monolayers.

Fundamental insight and technological applications have evolved. New phenomena and new materials emerge. Our understanding has been deepened for such diverse phenomena as melting in two dimensions, or the onset of turbulence in a Rayleigh-Benard experiment in $\mathrm{He}$. The dispersion of surface phonons and of electron energy bands has been determined experimentally for the first time. Wigner localization (in two dimensions) seems to have been observed (Fig. 1) and a superconducting state was discovered in a (quasi one-dimensional) organic conductor under a pressure of $12 \mathrm{kbar}$. A model to describe spin glasses seems to unfold and a "new" state of matter (Fig. 2) (involving bound vortex pairs with opposite vorticity in thin films of ${ }^{4} \mathrm{He}$ ) has been first predicted and then observed. Micro-electronics is one of the predominant fields of successful application; in "submicron devices", soon "man-made" quantum effects should be encountered. Other applications concern opto-electronics or solid state displays.

Condensed matter physics in an extremely vast discipline and emphasis is shifting rapidly as general conferences like the "March Meeting" of the American Physical Society or our own European Condensed Matter Division Conference succeed each other.

At the focus of attention at the 1980 APS March Meeting were photoemission spectroscopy as a probe of electronic band structure, surface probing via EXAFS, tunnelling two-level systems in metallic glasses, surface magnetism, superlattices - the newly designed close packing for computer memories - the physics of onedimensional organic conductors, $\mathrm{CuCl}$ and the diamagnetic anomaly, localization in thin wires and films, space charge layers and Wigner localization, intermediate valence etc. ...

Most of the topics dominating the APS Meeting were also central themes at the Antwerp meeting in April. Nevertheless, in Antwerp the emphasis was somewhat different as can be seen from the report in Europhysics News 11, (1980) 5, p. 7. The creation of a new quantum gas - atomic hydrogen (a little at the border of "condensed" matter) - and nuclear antiferromagnetism or ferromagnetism were two

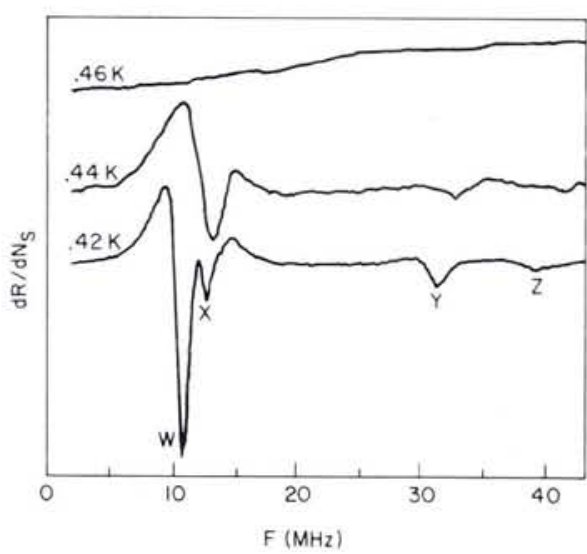

Fig. 1 - Experimental traces displaying the sudden appearance with decreasing temperature of coupled plasmon-ripplon resonances. The resonances only appear below $0.475 \mathrm{~K}$ where the sheet of electrons floating on a liquid helium surface has crystallized into a triangular lattice. (From C.C. Grimes and G. Adams, Phys. Rev. Lett., 42 (1980) 796)

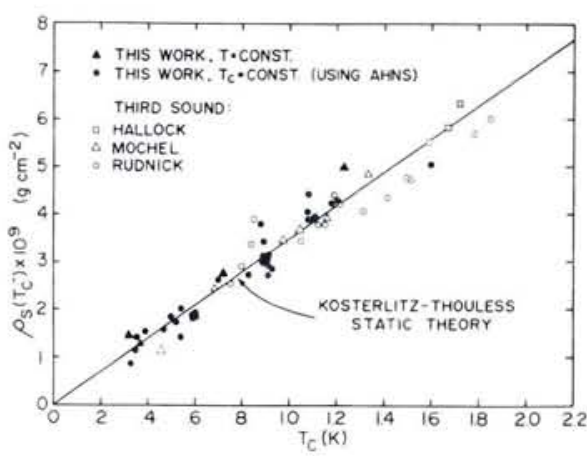

Fig. 2 - Results of the static jump in the superfluid density as a function of the transition temperature. Also included are previous third-sound results. The solid line is the Kosterlitz-Thouless static theory. (From D.J. Bishop and J.D. Reppy, Phys. Rev. Lett., 40 (1978) 1727) 